Supporting information:

\title{
Metadynamics in Essential Coordinates: Free Energy Simulation of Conformational Changes
}

\author{
Vojtěch Spiwok, Petra Lipovová, Blanka Králová
}

Contents:

Figure S1 .............................................. page 2

Figure S2 ................................................ page 3

Figure S3 ................................................. page 4

Figure S4 ............................................. page 5

Figure S5 ............................................... page 6 


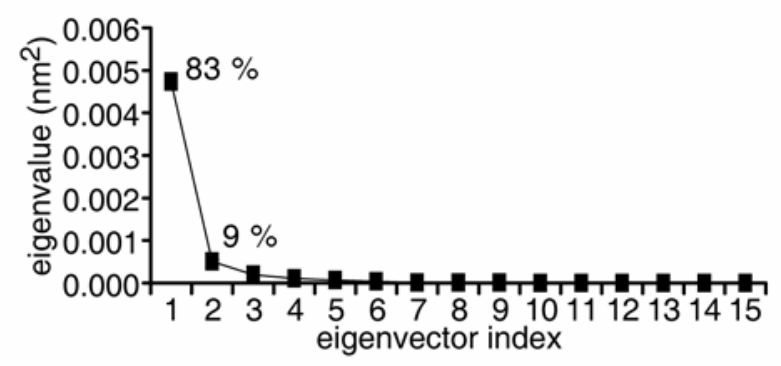

Figure S1. Eigenvalue plot (eigenvalue as a function of eigenvector index). This plot illustrates that the two most intensive collective motions account for the majority of overall motions of atoms selected for essential dynamics analysis ( $92 \%$ of the sum of eigenvalues). 
A
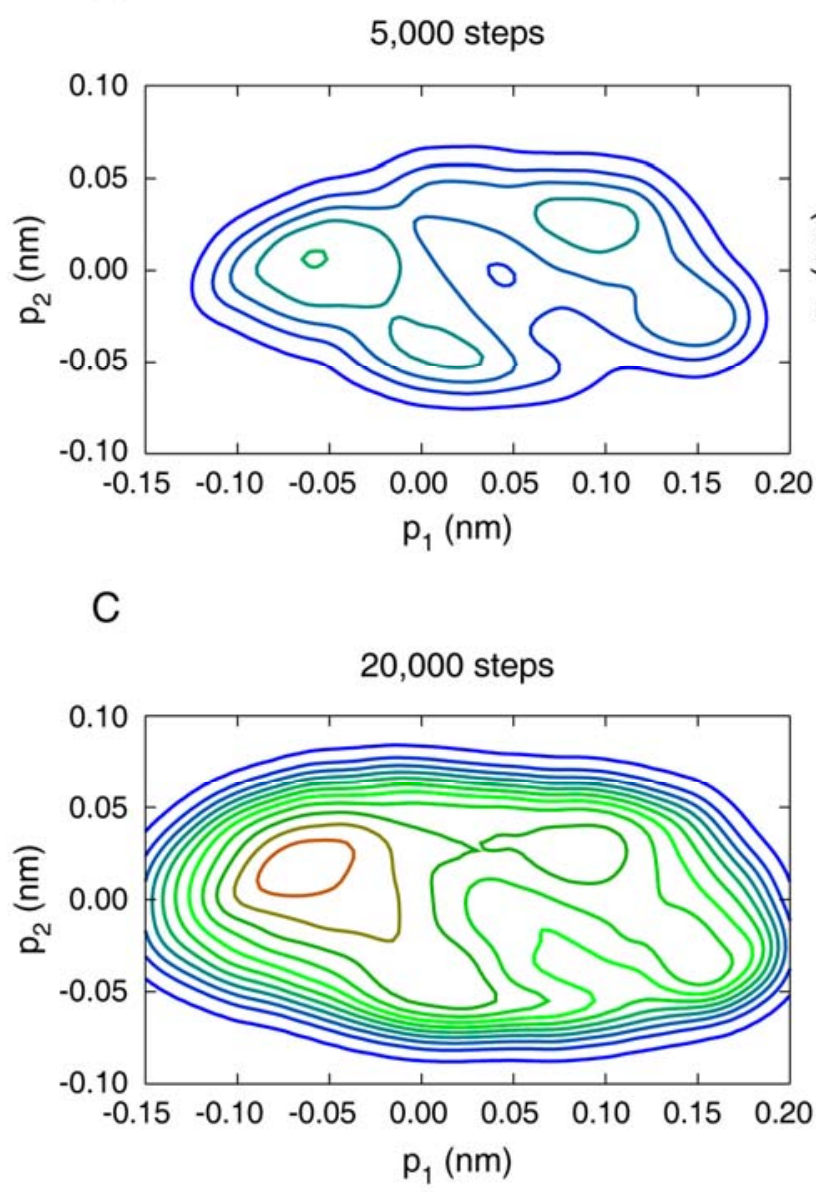

B
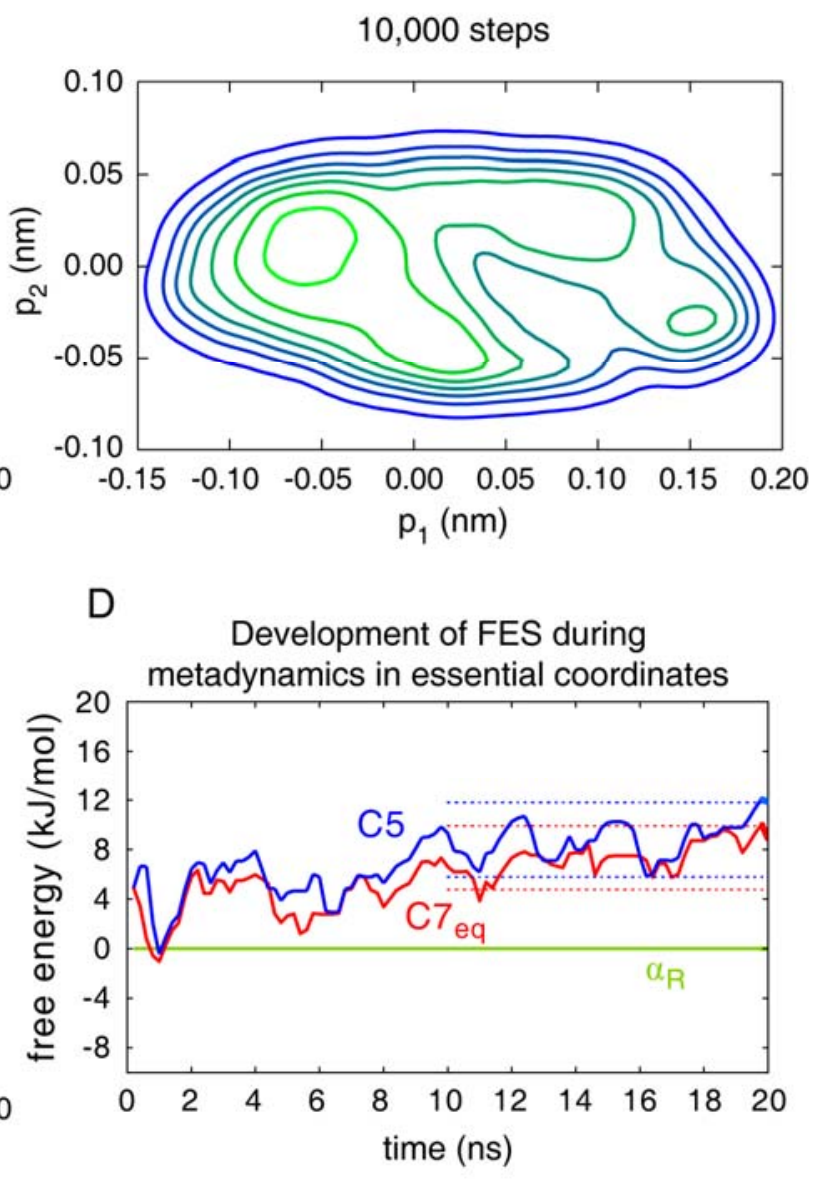

Figure S2: Development of the free energy surface as a function of essential coordinates during metadynamics in essential coordinates (starting from the $\alpha_{R}$ conformation). The free energy surface is illustrated after 5,000 (A), 10,000 (B) and 20,000 (C) steps. Contours are plotted every $4 \mathrm{~kJ} / \mathrm{mol}$. One step corresponds to $1.0 \mathrm{ps}$ of classical MDS (1.2 ps including equilibration phase). Local minima corresponding to $\alpha_{R}, C 7$ eq and C5 states were localized every 200 steps using a simple grid-based minima finder. Calculated values as a function of time are illustrated (D). Last 10,000 steps were used for calculation of average values and confidence intervals. 
A

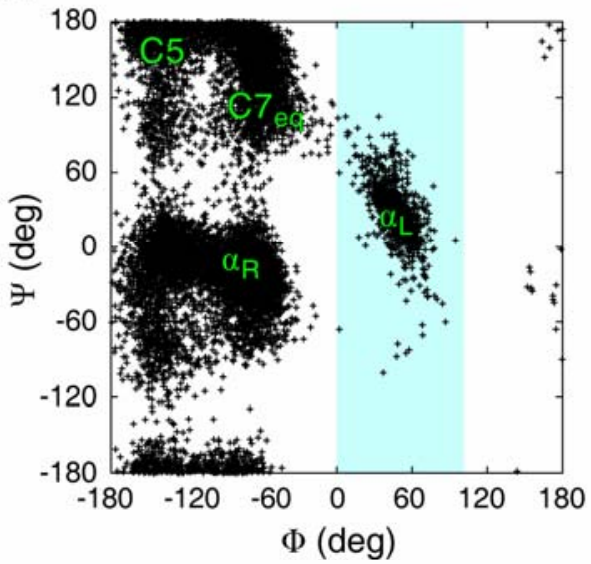

C

sum of Gaussians other than $\alpha_{L}$

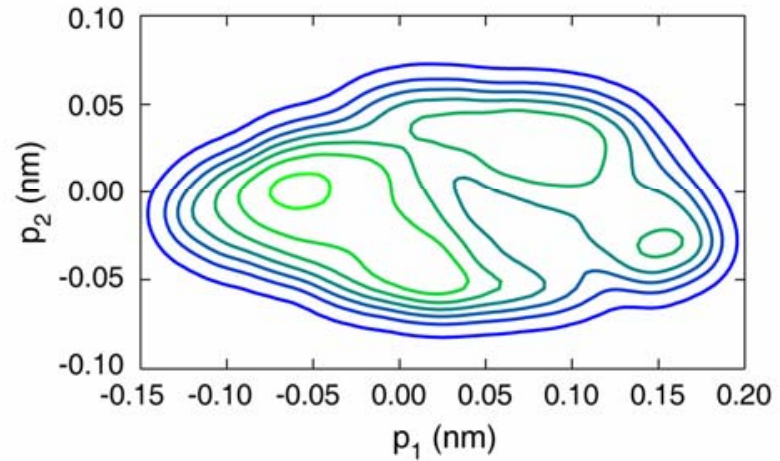

B

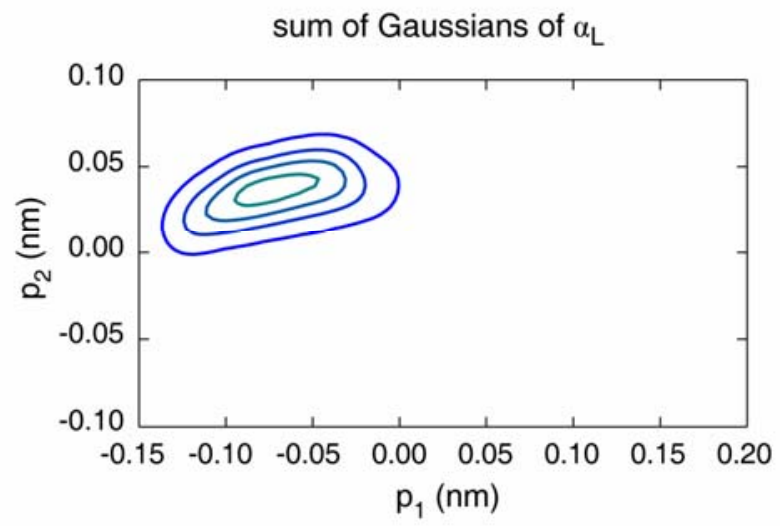

D

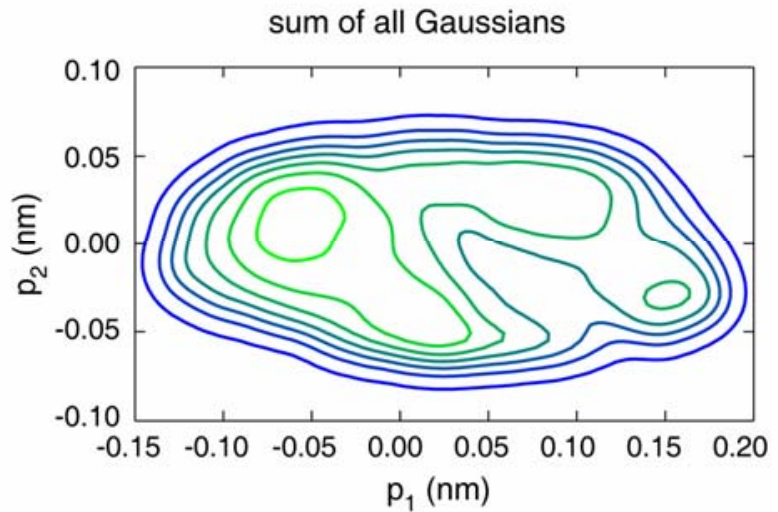

Figure S3: This figure addresses the problem of sampling the $\alpha_{\mathrm{L}}$ conformation. Essential coordinates of this conformation were overlying those of the $\alpha_{\mathrm{R}}$ conformation. In order to assess the effect of sampling of the $\alpha_{\mathrm{L}}$ conformation, dihedral angles $\Phi$ and $\Psi$ were calculated for initial coordinates of the first 10,000 steps of metadynamics (A). Steps with values $0 \mathrm{deg}<\Phi<100 \mathrm{deg}$ are located on the blue background. Sum of Gaussians corresponding to these steps is illustrated (B). Comparison of the free energy surface from which these Gaussians were subtracted (C) with the original free energy surface (D) indicates that an effect of sampling of $\alpha_{\mathrm{L}}$ conformation on free energy values is small (approx. $1.2 \mathrm{~kJ} / \mathrm{mol}$ for $\alpha_{\mathrm{R}}$ ). Contours are plotted every $4 \mathrm{~kJ} / \mathrm{mol}$. 
A

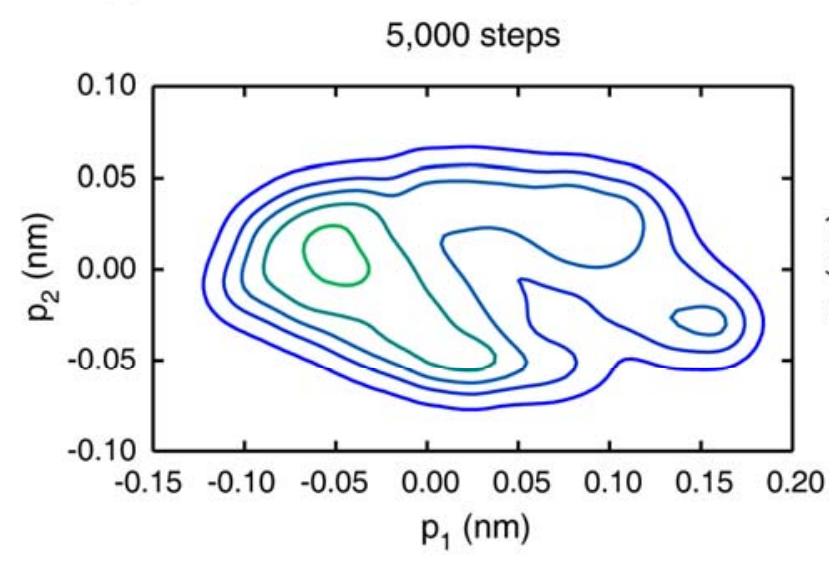

C

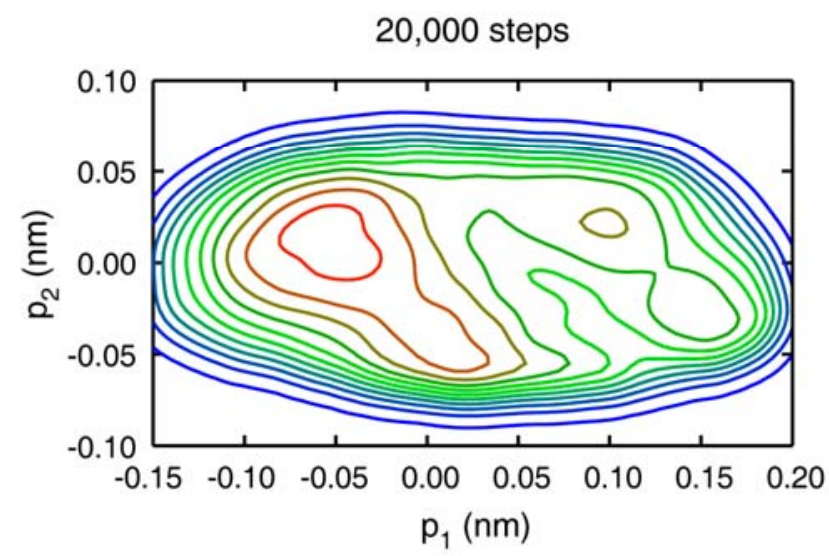

B

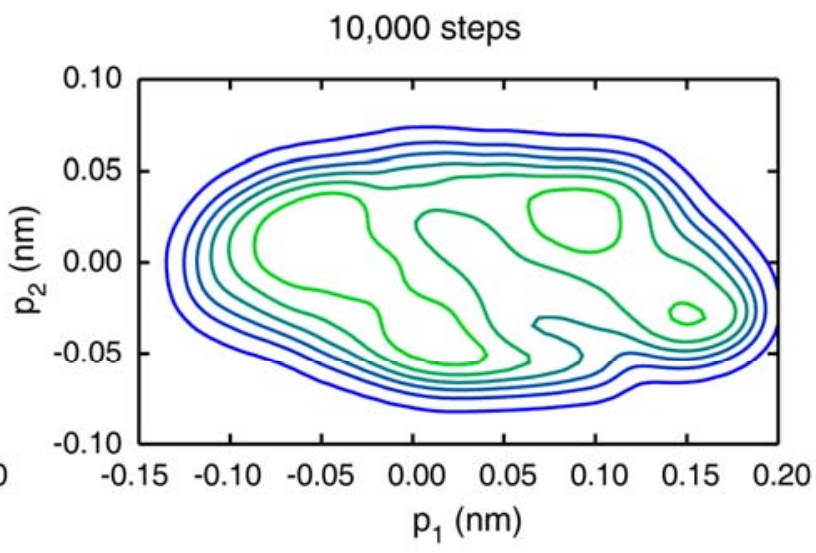

D

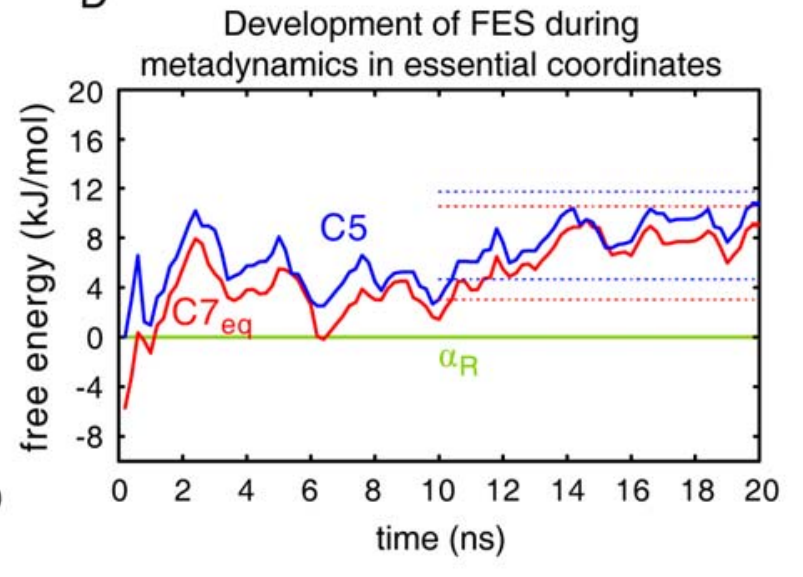

Figure S4: Accuracy of metadynamics in essential coordinates could be assessed by comparing it to another metadynamics run starting from a different initial state. The free energy surface, calculated by metadynamics in essential coordinates starting from $\mathrm{C}_{\mathrm{eq}} / \mathrm{C} 5$ conformation, is illustrated after 5,000 (A), 10,000 (B) and 20,000 (C) steps. Contours are plotted every $4 \mathrm{~kJ} / \mathrm{mol}$. Local minima corresponding to $\alpha_{R}$, $\mathrm{C}_{\text {eq }}$ and $\mathrm{C} 5$ states were localized as described in the caption of Figure S2 and their free energies as a function of time are illustrated (D). 


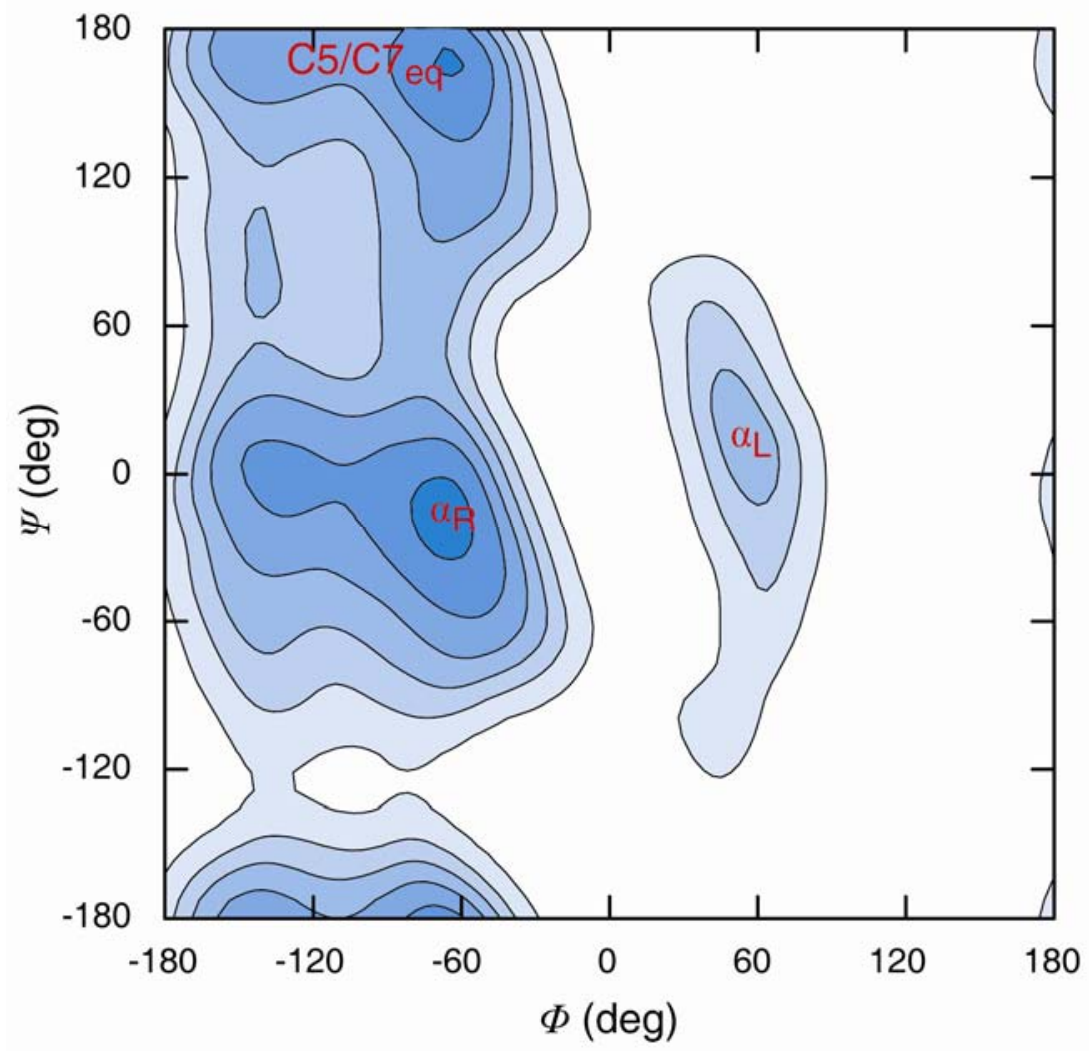

Figure S5: Accuracy of metadynamics in essential coordinates was also assessed by comparing it to metadynamics in the space of dihedrals $\Phi$ and $\Psi$. The free energy surface, calculated by metadynamics in dihedrals starting from $\alpha_{R}$ conformation is illustrated after 10,000 steps. Parameters used here were the same as in metadynamics in essential coordinates $(w=0.1 \mathrm{~kJ} / \mathrm{mol}$, microscopic MDS consisted of $0.2 \mathrm{ps}$ of equilibration and $1.0 \mathrm{ps}$ of sampling), except the parameter $\delta p$ which was $10 \mathrm{deg}$. Local minima corresponding to $\alpha_{\mathrm{R}}$ and $\mathrm{C} 5 / \mathrm{C} 7_{\text {eq }}$ states were localized as described in the caption of Figure S2. Free energy of the state $\mathrm{C} 5 / \mathrm{C} 7_{\text {eq }}$ was calculated as $5.6 \pm 2.3 \mathrm{~kJ} / \mathrm{mol}$. States $\mathrm{C} 5$ and $\mathrm{C} 7$ eq were not sufficiently resolved. Contours are plotted every $4 \mathrm{~kJ} / \mathrm{mol}$. 